\title{
Performance analysis of the thermoacoustic refrigerator with the standing wave and air as a working fluid
}

\author{
Jakub Kajurek ${ }^{1, *}$, and Artur Rusowicz ${ }^{1}$ \\ ${ }^{1}$ Warsaw University of Technology, Institute of Heat Engineering, Nowowiejska 21/25, 00-665 \\ Warsaw, Poland
}

\begin{abstract}
Thermoacoustic refrigerator is a new and emerging technology capable of transporting heat from a low-temperature source to a hightemperature source by utilizing the acoustic power input. These devices, operating without hazardous refrigerants and owning no moving components, show advantages of high reliability and environmental friendliness. However, simple to fabricate, the designing of thermoacoustic refrigerators is very challenging. This paper illustrates the impact of significant factors on the performance of the thermoacoustic refrigerator which was measured in terms of the temperature difference generated across the stack ends. The experimental device driven by a commercial loudspeaker and air at atmospheric pressure as a working fluid was examined under various resonator length and operating frequencies. The results indicate that appropriate resonator's length and operating frequency lead to an increase in the temperature difference created across the stack. The maximum values were achieved for operating frequency equalled to 200 and $300 \mathrm{~Hz}$ whereas resonator length corresponded to the half-length of the acoustic wave for these frequencies. The results of experiment also confirm that relationship between these parameters is strongly affected by the stack spacing, which in this research was equalled to $0.4 \mathrm{~mm}$.
\end{abstract}

\section{Introduction}

Thermoacoustic refers to all processes in which acoustic energy is converted into heat and vice versa. As a result, during last three decades we could observe a rapid advancement in thermoacoustic technology such as development of thermoacoustic theory together with the prototype devices both engines and refrigerators $[1,2]$. The thermoacoustic engines generate acoustic power by utilizing heat flowing from a high-temperature source to a low-temperature source, while the thermoacoustic refrigerators absorb acoustic power to transfer heat from a low-temperature source to a high-temperature source. All thermoacoustic devices are attractive not only from environmental but also fabricating and maintaining perspective. In contrast to traditional devices they have no moving components or even sliding seals and do not use hazardous refrigerants or chemicals [3-5]. Nevertheless, the applications of the

\footnotetext{
* Corresponding author: jakub.kajurek@itc.pw.edu.pl
} 
thermoacoustic devices are limited due to lower performance in comparison to commercial solutions. For example the thermoacoustic refrigerators achieve 10 to $20 \%$ of Carnot efficiency as opposed to 33 to $50 \%$ for vapour-compression refrigerators [6].

In the thermoacoustic refrigerators the acoustic energy can be provided by a thermoacoustic engine or by an acoustic driver e.g. a loudspeaker. A schematic illustration of a standing-wave thermoacoustic refrigerator driven by an acoustic driver is shown in Fig. 1a. The device consists of an acoustic driver, a resonator, a stack, the heat exchangers and a working fluid. The fluid parcels inside the resonator are compressed and expanded adiabatically by an acoustic standing wave sustaining by the acoustic driver at the fundamental resonance frequency for the resonator. For the fluid parcels oscillating within the stack, at about a thermal penetration depth from the plates, the thermal interaction with the plates modifies original temperature oscillations caused by the acoustic wave, both in the magnitude and the phase. The positional displacement of the fluid parcel toward the pressure antinode is accompanied with an increase in pressure and temperature in the wave and a heat transfer from the fluid to the plate (Fig. 1b). The subsequent rarefaction of the fluid parcel results in a decrease in pressure and temperature and then in a heat transfer back to the fluid from the plate [7]. Since there are many fluid parcels oscillating at the distance of the thermal penetration depth from the plate along the stack, heat transferred by one fluid parcel is deposited on the plate and then the same amount of heat is further transported by the adjacent parcel [8]. Hence the temperature difference $\Delta T$ develops along the stack plates (Fig. 1c). In order to exploit this effect for heat pumping process in the refrigerator, the heat exchangers have to be attached at both ends of the stack. The cold heat exchanger transfers heat from a low-temperature source to cold end of the stack, while the hot heat exchanger extracts heat from hot end of the stack to a high-temperature source. A detailed explanation of how thermoacoustic devices works is given by Swift [7].

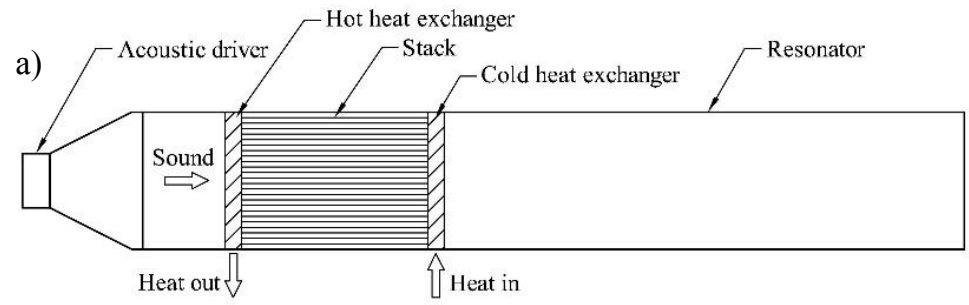

b)

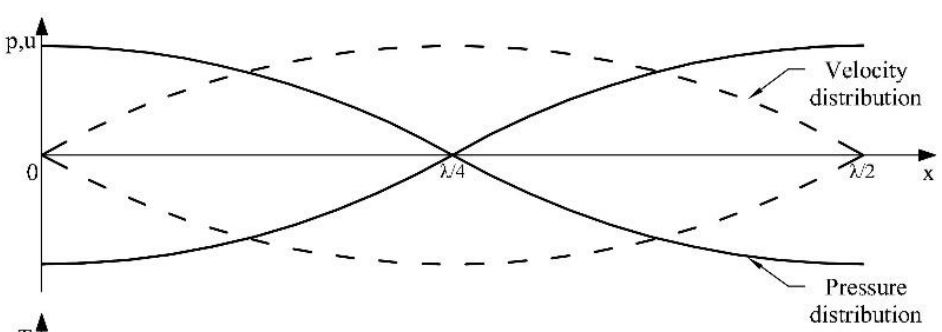

c)

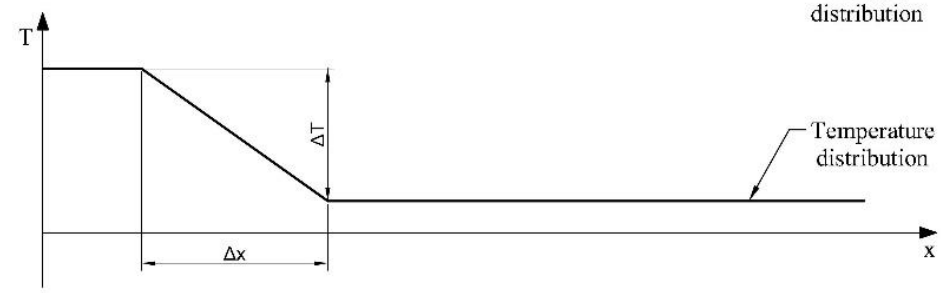

Fig. 1. a) Schematic illustration of the standing-wave thermoacoustic refrigerator with an acoustic driver. b) Pressure and velocity distribution in resonator. c) Temperature distribution in resonator. 
The performance of the thermoacoustic refrigerator with the standing wave driven by an acoustic driver is influenced by many factors, such as: geometrical parameters (like stack length, stack porosity or stack centre position), material specific parameters (like thermal conductivity, density and specific heat of working fluid and stack material) and operating parameters (like mean temperature and pressure, resonance frequency or cooling load) [8]. Recently many efforts have been taken to optimize the devices operation. They are extensively studied theoretically as well as experimentally. Most of the research have been done on the stack [9]. Tijani et al. [10] investigated the optimal stack spacing for parallelplate geometry. According to their results the desired separation gap between the plate should be between 2 and 4 thermal penetration depths. Other structures of the stack, like spiral, honeycomb and corning comb were also experimentally tested. In [11] the effect of stack material, geometry, length and position on temperature difference generated across the stack ends was studied. The maximum temperature difference was achieved for $4 \mathrm{~cm}$ Corning Celor stack located $4 \mathrm{~cm}$ from sound source. Many researches were done only on stack location as well. The optimum stack position was measured in terms of temperature difference $[12,13]$ or in terms of COP [14]. Most often the best location falls between $\lambda / 20$ and $\lambda / 8$, where $\lambda$ is the wavelength [9]. Another experiments concerns operating frequency and resonator length. Wantha et al. [15] investigated the impact of the resonance tube on the performance of the thermoacoustic stack. They found that the optimal operating frequency differs from the design based on equation $f_{0}=a /\left(2 L_{0}\right)$ and that the appropriate combination of the operating frequency and the resonance tube's length lead to an increase in temperature difference by approximately $57 \%$. Also Tang et al. [16] showed that the resonator length has significant influence on the resonance frequency, which in their measurements decreased with the increase of the resonance tube's length.

The study presented in this paper investigate the effect of the operating frequencies and resonance tube length on the performance of the thermoacoustic refrigerator. A prototype device driven by a loudspeaker and with air as a working fluid was assembled for experimentation within mentioned parameters. The cold and heat exchangers were removed from experimental apparatus. The thermoacoustic effect was measured only in terms of the temperature difference generated across the stack.

\section{Experimental apparatus and procedure}

Fig. 2 illustrates the arrangement of experimental apparatus. The apparatus was only used to measure the temperature difference created between the stack ends and thus the thermoacoustic device did not have hot and cold heat exchanger. A commercial loudspeaker with $200 \mathrm{~W}$ continuous power was used as an acoustic driver. It was attached to the one end of the rectangular resonance tube with inner dimensions $45 \times 45 \mathrm{~mm}$, filled with air at atmospheric pressure. The second end of the resonator was closed tightly by a movable piston, which allowed to change the resonance tube length. The parallel-plate stack installed inside the resonator was made from PET plates with $0.178 \mathrm{~mm}$ thickness and $5 \mathrm{~cm}$ length. The plates were spaced by fishing line with $0.4 \mathrm{~mm}$ thickness. An audio generator, installed on the computer was used to produce the acoustic signal with determined frequency and intensity that was transferred to the amplifier. The amplified signal excited the loudspeaker, which sustained an acoustic wave in the resonator. The temperature difference created across the stack due to interactions between the working fluid and stack plates was measured by the thermocouple type-T connected to data acquisition card. The temperature value for each end of the stack was displayed by the special program installed on the computer with the accuracy $0.1 \mathrm{~K}$. Taking into account all important parameters which influence on the measurement, the overall accuracy of the experimental data is of the order of $10 \%$. 


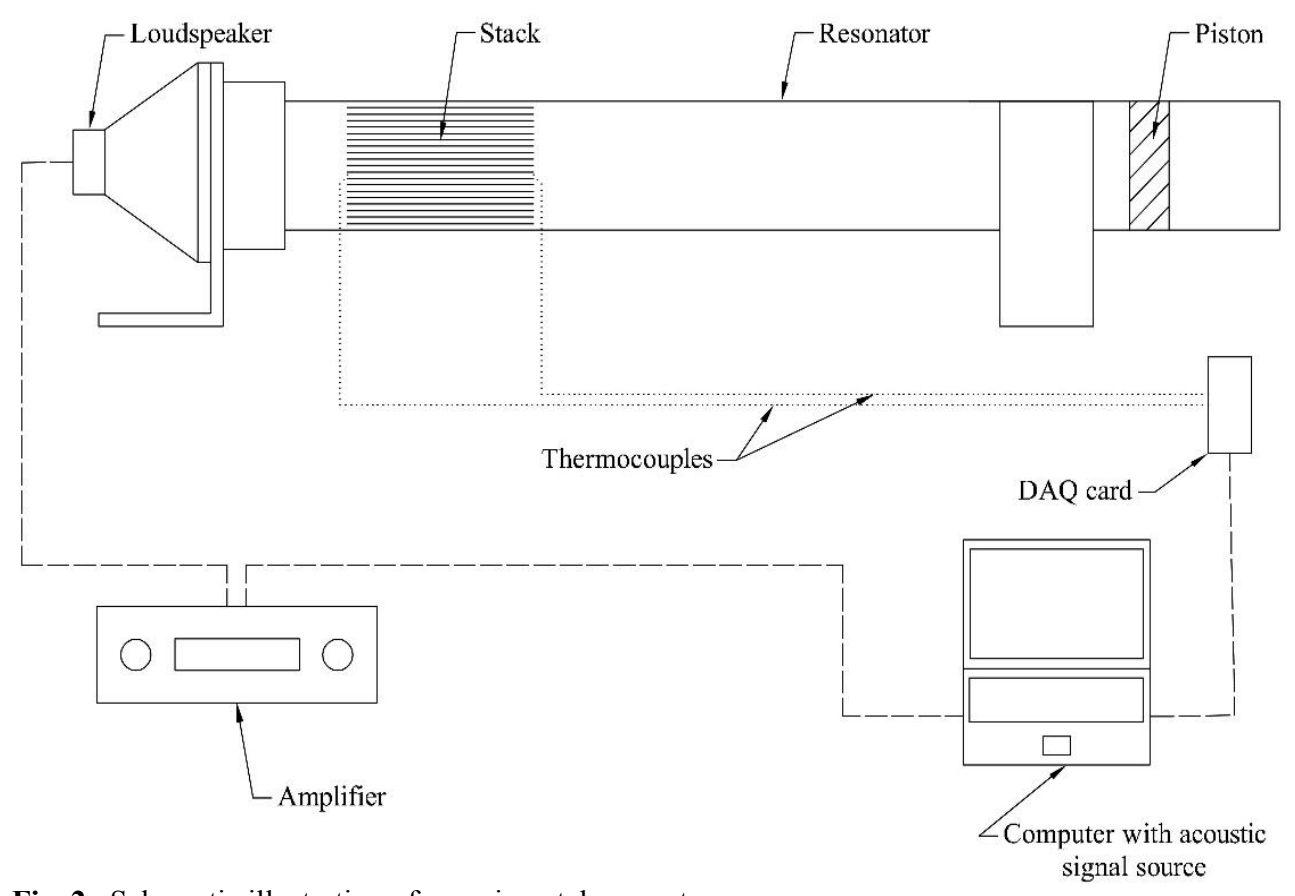

Fig. 2. Schematic illustration of experimental apparatus.

The experiments were conducted to evaluate the performance of the thermoacoustic refrigerator under various operating frequencies and resonator length. The resonator lengths were chosen to match the half-wavelength, which is defined as

$$
f_{0}=\frac{a}{2 L_{0}}
$$

where $f_{0}$ is the resonance frequency, $L_{0}$ is the length of the resonator and $a$ is the sound's speed. The resonator's length was estimated for five different resonance frequencies and was equalled to: $L_{1}=1.71 \mathrm{~m}$ for $100 \mathrm{~Hz}, L_{2}=0.855 \mathrm{~m}$ for $200 \mathrm{~Hz}, L_{3}=0.57 \mathrm{~m}$ for $300 \mathrm{~Hz}$, $L_{4}=0.428 \mathrm{~m}$ for $400 \mathrm{~Hz}$ and $L_{5}=0.342 \mathrm{~m}$ for $500 \mathrm{~Hz}$. In every measurement the stack was placed at $1 / 8$ of the resonator's length. The position corresponded also to $1 / 16$ of the acoustic wave length for which the length of the resonator was calculated. For given resonator's length 21 operating frequencies varying from 100 to $500 \mathrm{~Hz}$ with $20 \mathrm{~Hz}$ step, at the same amplification $(-9 \mathrm{~dB})$ were examined in terms of the temperature difference created across the stack. For each measurement the data was read until the temperature difference between the stack ends became stable. After running the experiments for the desired frequency range, the resonator's length and stack position were adjusted to new values and the investigation was repeated.

\section{Experimental results and discussion}

The temperature differences generated between the stack ends versus operating frequencies and resonator's lengths are shown in Fig. 3. The graphs look like a sinusoid with the highest temperature difference at the resonance frequency. The highest values, equalled to 13.6 and $12.6 \mathrm{~K}$, were achieved at 200 and $300 \mathrm{~Hz}$ respectively, and resonator's length corresponded to the half-length of acoustic wave for these frequencies. The results suggest that optimal configuration should be somewhere between these two. For the longest resonance tube (L1) 
the highest value (about $7 \mathrm{~K}$ ) was not achieved for the first resonance frequency, but for the second - $200 \mathrm{~Hz}$. The difference is small and might have been caused by the difference in the thermal penetration depth between these two frequencies. For the fifth resonator's length (L5) the highest temperature difference also occurred at two frequencies 400 and $500 \mathrm{~Hz}$ and was equalled to 7.2 and $7.9 \mathrm{~K}$ respectively. At $400 \mathrm{~Hz}$ and resonance tube length $\mathrm{L} 4$ the temperature difference was equalled to $9.4 \mathrm{~K}$.

For each resonator's length the temperature difference increases continuously with operating frequency. Further variation in the frequency causes the decrease in the temperature difference. The differences in temperatures are caused by mutual modification of the incident and reflected wave and due to the change in the thermal and vicious penetration depth. A high operating frequency leads to a low thermal penetration depth and thus not all of the working fluid contained between plates might have taken part in heat transfer when the plate spacing was too wide. On the other hand, low operating frequencies causes increase in the thermal penetration depth, so the heat pumping process might have been affected by the plate temperature when the plate spacing was too narrow. Also the stack position influences the temperature difference. In the experiment the stack was located at $1 / 8$ of the resonator's length. However, from the acoustic wave point of view the stack location changed with the change of the operating frequency. The temperature difference decreases for the stack positions closer to the pressure or velocity node. A zero value of the temperature differences means that the stack is in the pressure or in the velocity node - point at which heat transfer vanishes [7]. A negative temperature differences arises from the change in heat transfer direction, the hot end of the stack becomes a cold end and the cold end becomes a hot end of the stack.

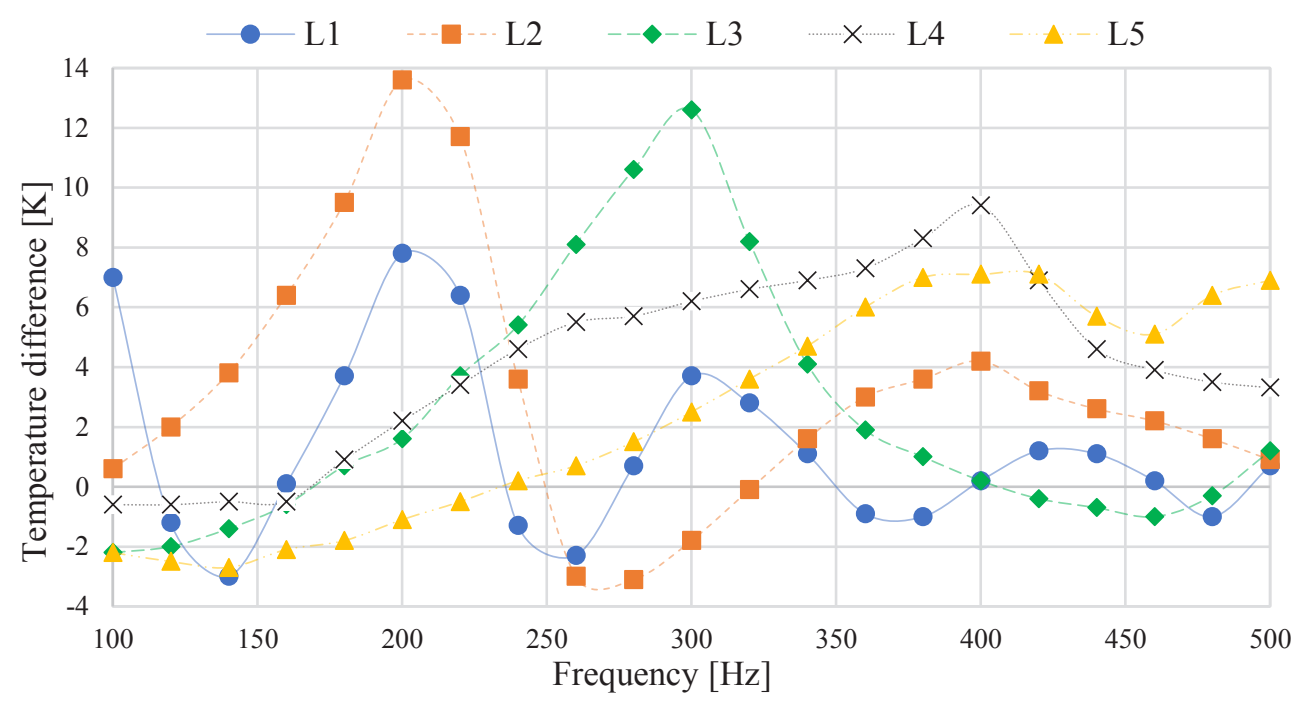

Fig. 3. Temperature difference between stack ends versus operating frequency for different resonator's length.

Fig. 4 presents an experimental results of the temperature evolution in time at hot and cold end of the stack for five frequencies and resonator's lengths equalled to the half-length of acoustic wave for these frequencies. As the acoustic power is supplied the heat starts to flow out of one end of the stack and flow into the other end. Hence the temperature of the end that supplies heat decreases and the temperature of the other end, which absorbs heat, increases. Fig. 5 shows that for all frequencies the temperature difference generated across 
the stack during first $50 \mathrm{~s}$ increases rapidly. After that time temperature curves becomes flat. This is because a diffusive heat flow through the stack driven by the temperature difference tends to balance a hydrodynamic heat flow. The diffusive heat flux is minimum at the beginning, when there is no temperature difference across the stack and when the hydrodynamic heat flux is maximum. The thermoacoustic heat transport diminishes as the temperature gradient develops. A steady state is reached when the heat transported due to the thermoacoustic effect is balanced by the diffusive heat flow in the gas and in the stack. From Fig. 4 a higher temperature increase at the hot end of the stack than the temperature decrease at the other end can be observed. The hold and cold end temperature profiles are not symmetric with the respect to the initial temperature. The experimental device has no heat exchangers and thus no heat is removed. An absorbed acoustic energy appears as a dissipative heat which is accumulated at the hot end of the stack, increasing the temperature at this point.

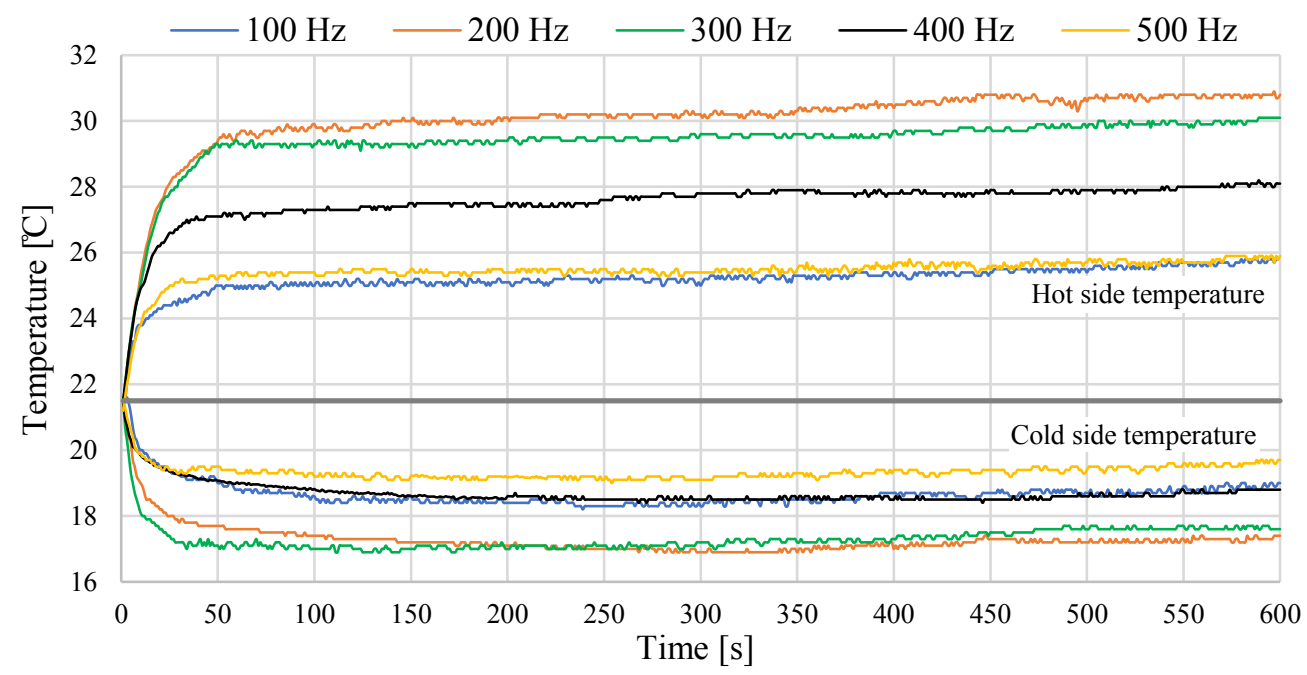

Fig. 4. Temperature evolution in time at the stack's ends.

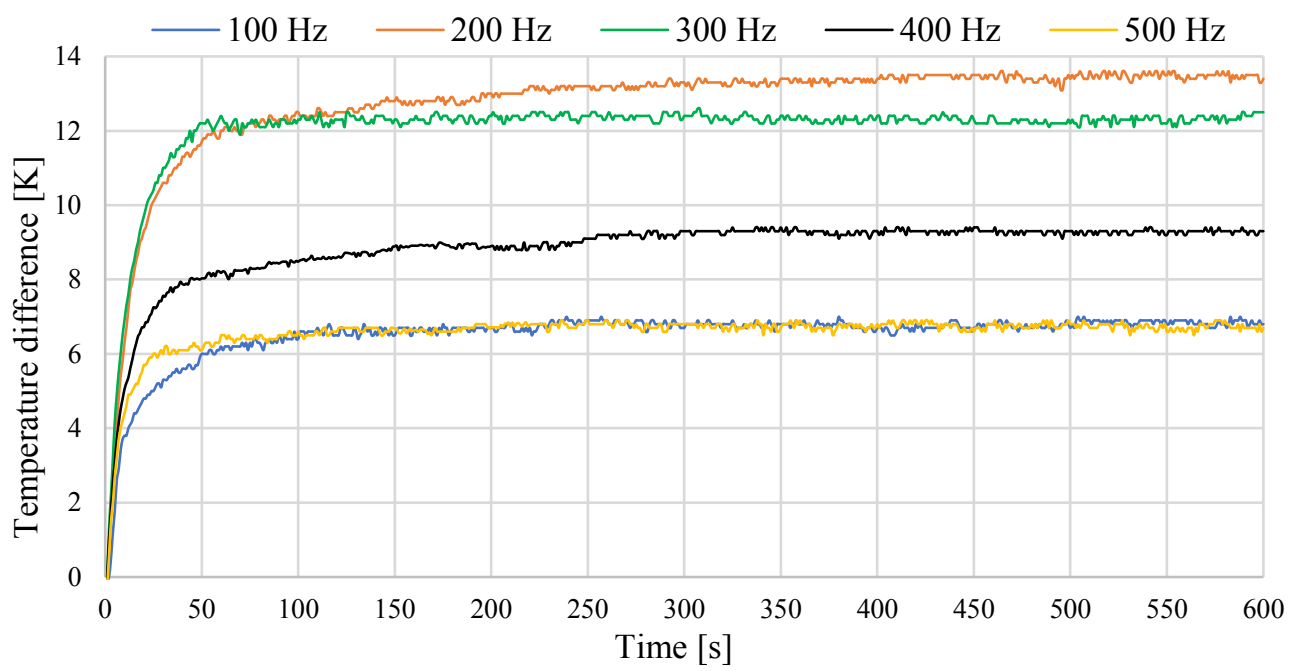

Fig. 5. Temperature difference evolution in time between the stack ends. 


\section{Conclusion}

In this study the influence of the operating frequency and resonance tube length on the performance of the standing wave thermoacoustic refrigerator with air as a working fluid were studied. Five different resonator's lengths at 21 operating frequencies varying from 100 to $500 \mathrm{~Hz}$ with $20 \mathrm{~Hz}$ step were examined in terms of temperature difference created between the stack ends.

The experiments confirm that the resonator length and operating frequency have significant impact on the operation of the thermoacoustic refrigerator. The appropriate parameters lead to an increase in the temperature difference generated across the stack. However, the plate spacing in the stack has to be also considered during the final selection. A poor match will cause a decline in stack performance. The results show also that the highest temperature differences are achieved for resonance frequencies. The temperature difference increases rapidly after the acoustic power is supplied and becomes constant when the thermoacoustic heat flow is balanced by the diffusive heat flow in the gas and in the stack. The results and conclusions are in good agreement with the existing literature. In this research the best operating parameters are 200 and $300 \mathrm{~Hz}$ with the half-wavelength resonator as they gave 13.6 and $12.6 \mathrm{~K}$ of the temperature difference between the stack ends respectively.

\section{References}

1. H. Babaei, K. Siddiqui, Energy Convers. Manag. 49, 14 (2008)

2. A. Grzebielec, A. Rusowicz, Rynek Energii 106, 6 (2013)

3. A. Ruciński, A. Rusoiwcz, A. Grzebielec, Gas engine driven heat pump characteristics, analysis of applications in buildings energy systems, $9 \mathrm{~h}$ International Conference Environmental Engineering (9th ICEE) - Selected papers

4. J. Oboleiwcz, Inżynieria Bezpieczeństwa 2, 11 (2017)

5. A. Grzebielec, Apar. Badaw. i Dydakt. 22, 6 (2017)

6. M. Wetzel, C. Herman, Int. J. Refrig. 20, 19 (1997)

7. G.W. Swift, J. Acoust. Soc. Am. 84, 36 (1988)

8. C. Herman, Z. Travnicek, Heat Mass Transf. Und Stoffuebertragung. 42, 9 (2006)

9. N.A. Zolpakar, N. Mohd-Ghazali, M. Hassan El-Fawal, Renew. Sustain. Energy Rev. 54, 9 (2016)

10. M.E.H. Tijani, J.C.H. Zeegers, A.T.A.M. de Waele, J. Acoust. Soc. Am. 112, 6 (2002)

11. S.H. Tasnim, S. Mahmud, R.A. Fraser, Heat Mass Transf. Und Stoffuebertragung. 48, 15 (2012)

12. M. Akhavanbazaz, M.H.K. Siddiqui, R.B. Bhat, Exp. Therm. Fluid Sci. 32, 9 (2007)

13. J. Kajurek, A. Rusowicz, A. Grzebielec, Arch. Thermodyn. 38, 19 (2017)

14. L.K. Tartibu, Heat Mass Transf. Und Stoffuebertragung. 52, 8 (2016)

15. C. Wantha, K. Assawamartbunlue, Front. Heat Mass Transf. 2, 8 (2011)

16. K. Tang, G.B. Chen, T. Jin, R. Bao, B. Kong, L.M. Qiu, Cryogenics (Guildf). 45, 7 (2005) 